\title{
Metallic Glacial Glass Formation by a First-order Liquid-liquid Transition
}

J. Shen ${ }^{1,2}$, Z. Lu ${ }^{3}$, J. Q. Wang ${ }^{2,4}$, S. Lan ${ }^{5,6}$, F. Zhang ${ }^{7}$, A. Hirata ${ }^{7}$, M.W. Chen ${ }^{8}$, X. L. Wang $^{6,9,10}$, P. Wen ${ }^{1,2,11}$, Y. H. Sun*1,2, H. Y. Bai*1,11,12 and W. H. Wang*1,2,11

1. Institute of Physics, Chinese Academy of Sciences, Beijing, 100190, China

2. School of Physical Sciences, University of Chinese Academy of Sciences, Beijing, 100049, China

3. Mathematics for Advanced Materials - Open Innovation Laboratory (MathAM-OIL), AIST, Sendai, 980-8577, Japan

4. Ningbo Institute of Materials Technology and Engineering, Chinese Academy of Sciences, Ningbo, 315201, China

5. Herbert Gleiter Institute of Nanoscience, Nanjing University of Science and Technology, Nanjing, 210094, China

6. Department of Physics and Materials Science, City University of Hong Kong, Hong Kong, China

7. WPI- Advanced Institute for Materials Research (WPI-AIMR), Tohoku University Sendai 980-8577, Japan

8. Department of Materials Science and Engineering, Johns Hopkins University, Baltimore, MD 21218, USA

9. City University of Hong Kong Shenzhen Research Institute, Shenzhen 518057, China

10. Center for Advanced Structural Materials, City University of Hong Kong, Hong Kong China

11. Songshan Lake Materials Laboratory, Dongguan, Guangdong, 523808, China

12. Center of Materials Science and Optoelectronics Engineering, University of Chinese Academy of Sciences, Beijing, 100049, China

Correspondence and requests for materials should be addressed to Y.H.S (ysun58@iphy.ac.cn), H.Y.B (hybai@iphy.ac.cn) and W.H.W (whw@iphy.ac.cn) 
Metallic glass samples. A master alloy was prepared by arc-melting the high-purity elements, including La (99.5\%), Ce (99.5\%), Co (99.98\%) and Al (99.99\%), under a Ti-gettered argon atmosphere. Rods, sheets, and ribbons were made according to the experiments. In particular, $1 \mathrm{~mm}$-thick plates and $2 \mathrm{~mm}$-in-diameter rods were made by suction-casting into a water-cooled $\mathrm{Cu}$-mould under a Ti-gettered argon atmosphere. Ribbons with a thickness of $\sim 35 \mu \mathrm{m}$ were prepared by melt-spinning molten liquid at $1050 \mathrm{~K}$ onto a copper wheel at a rotation speed of $60 \mathrm{~m} / \mathrm{s}$ in an argon atmosphere. The annealed (or the transformed) samples were prepared by heating the as-cast samples up to the end temperature of the first post- $T_{\mathrm{g}}$ exothermic peak at $10 \mathrm{~K} \mathrm{~min}{ }^{-1}$ and then immediately cooled down to room temperature at $100 \mathrm{~K} \mathrm{~min}^{-1}$ at argon atmosphere.

Differential scanning calorimetry (DSC) was performed on Perkin-Elmer DSC 8000 calorimeter. The ribbons or discs cut from rods and sheets were placed in a standard aluminum crucible and protected under a flow of high-purity argon gas. The applied heating and cooling rates are $10 \mathrm{~K} \mathrm{~min}^{-1}$ and $100 \mathrm{~K} \mathrm{~min}^{-1}$ respectively. The absolute value of the specific heat capacity was calibrated by the reference of a sapphire standard whose specific heat capacity is documented.

In-situ synchrotron X-ray scattering measurements were performed at station 11-IDC of the Advanced Photon Source, Argonne National Laboratory, using a $105.721 \pm$ $0.006 \mathrm{keV}$ x-ray beam. The $35 \mu \mathrm{m}$-thick thin ribbons of the melt-spun and $480 \mathrm{~K}$ annealed $\mathrm{La}_{32.5} \mathrm{Ce}_{32.5} \mathrm{Co}_{25} \mathrm{Al}_{10}$ were mounted in a Linkam TS 1500 furnace for thermal scanning. The incident $\mathrm{x}$-ray beam, defined by the upstream slits, was $0.5 \mathrm{~mm}$ wide by $0.5 \mathrm{~mm}$ high for these measurements. The data acquisition time for each pattern is $1 \mathrm{~s}$ yet the total acquisition time is $\sim 6 \mathrm{~s}$, including the time for data saving. The heating rate is maintained at $10 \mathrm{~K} \mathrm{~min}^{-1}$ (for $\mathrm{La}_{32.5} \mathrm{Ce}_{32.5} \mathrm{Co}_{25} \mathrm{Al}_{10}$ ), and the temperature precision is $\pm 1 \mathrm{~K}$. The total structural factor $S(q)$ with $q_{\max } \sim 15 \AA^{-1}$ was derived from the scattering data by integrating images, subtracting the appropriate background and correcting for fluorescence, polarization, absorption, multiple scattering and inelastic scattering using the Amorphous Scattering Code written by Prof. T. C. Hufnagel.

Transmission electron microscopy (TEM): The TEM specimens were carefully prepared by ion-milling (Gatan PIPS II 695) with $3 \mathrm{keV}$ Ar ions and $0.5 \mathrm{keV}$ for finally 
milling at liquid nitrogen temperature. The high-resolution TEM (HRTEM) and high angle annular dark field-scanniTEM (HAADF-STEM) were performed on a cold field emission TEM (JEOL, JEM-2100F TEM/STEM) with double spherical aberration (Cs) correctors (operated at $200 \mathrm{kV}$ ). The scanning NBED is performed by utilizing an electron beam with an FWHM diameter of 3-5 $\mathrm{nm}$.

Vicker's micro-hardness $\left(\boldsymbol{H}_{\mathbf{v}}\right)$ was performed via an HENGYi MH-51 indenter. An indentation load of $0.5 \mathrm{~kg}$ was applied. One as-cast $\mathrm{La}_{32.5} \mathrm{Ce}_{32.5} \mathrm{Co}_{25} \mathrm{Al}_{10}$ plate was indented and repeatedly heated up to different $T_{\mathrm{AS}}$, i.e. $T_{\mathrm{A}}=353-576 \mathrm{~K}$, cooled to room temperature and indented to get $H$ v. 20 indentations were made at each experimental condition.

Fast Differential Scanning Calorimetry (FDSC) was examined on Flash DSC2+ (METTLER TOLEDO). A $0.2 \sim 1 \mu \mathrm{g} \mathrm{La}_{32.5} \mathrm{Ce}_{32.5} \mathrm{Co}_{25} \mathrm{Al}_{10}$ specimen was carefully cut from a ribbon under an optical microscope. The specimen was then transferred to the sample chip before FDSC experiments. All the measurements were performed under an argon atmosphere using the same sample chip. Heating rates were covered from $50 \mathrm{~K}$ $\mathrm{s}^{-1}$ to $5000 \mathrm{~K} \mathrm{~s}^{-1}$ and the temperature range was from $298 \mathrm{~K}$ to $773 \mathrm{~K}$. 

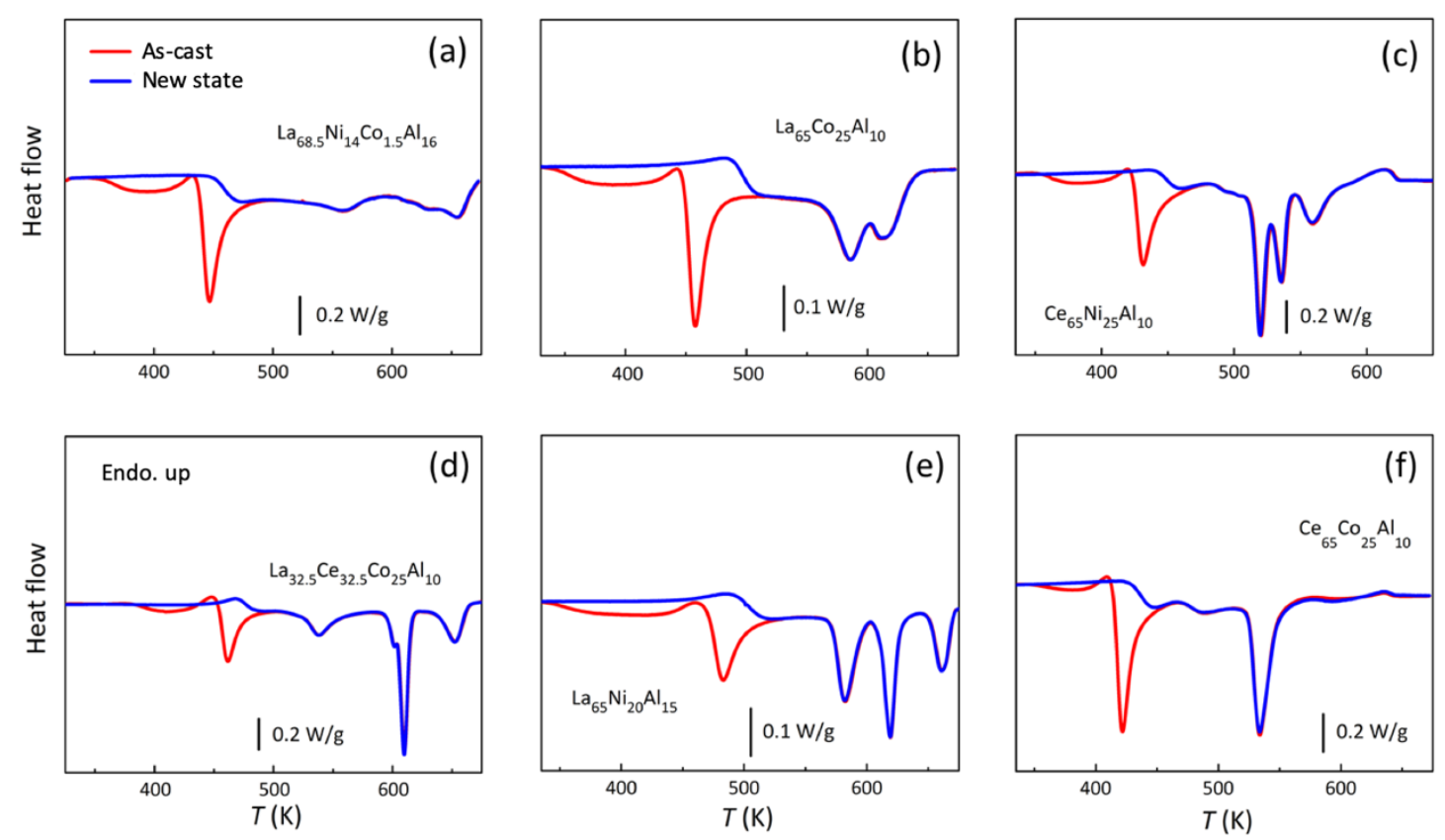

Figure S1. DSC for the as-cast MG (red curve) and the new state (blue curve) of six different compositions. A series of $\mathrm{La} / \mathrm{Ce}$-based MG compositions that have the similar features of exothermic peaks after glass transition, and the new state are developed using the same method as in Figure 1, demonstrating the universality of the first-order transition in these RE-TM-Al MGs. All the DSC curves are measured at a heating rate of $20 \mathrm{~K} \mathrm{~min}^{-1}$. After the transformation, all the samples are confirmed to be amorphous by laboratory XRD. 


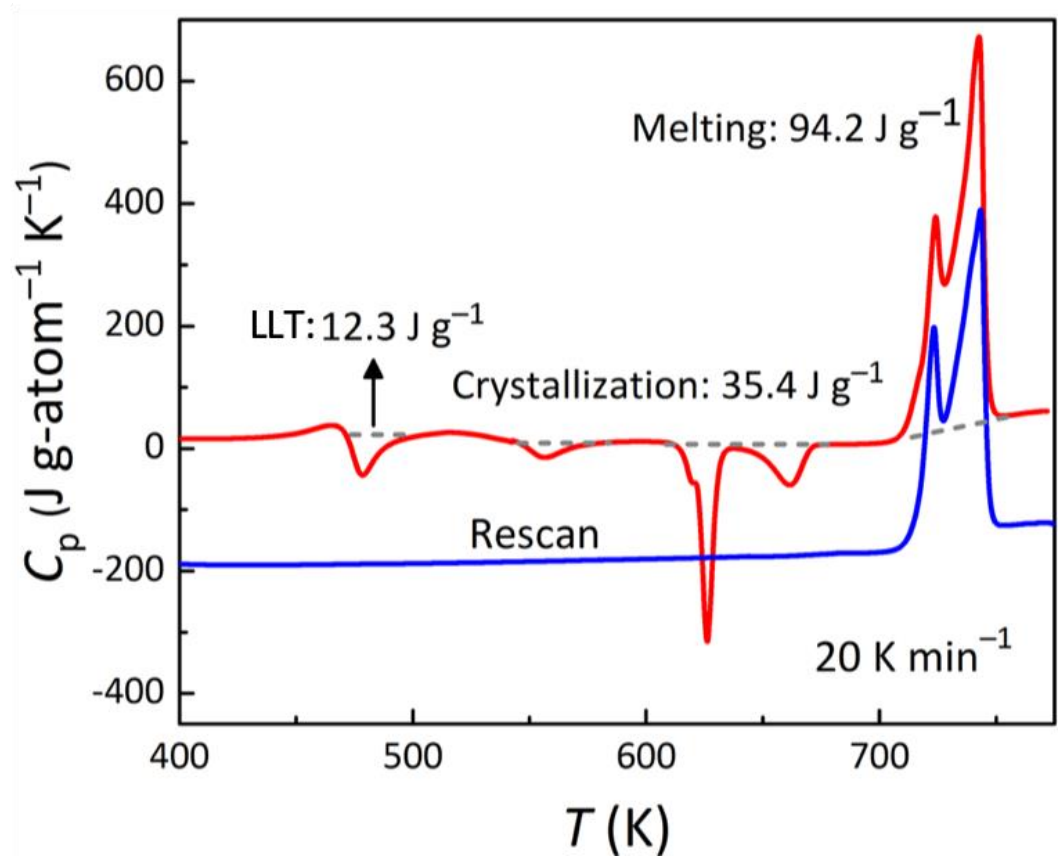

Figure S2. The DSC curves of an as-cast La-based MG and the fully crystalline at a heating rate of $20 \mathrm{~K} \mathrm{~min}^{-1}$. The enthalpy of the exothermic and endothermic events are calculated from the enclosed area. 


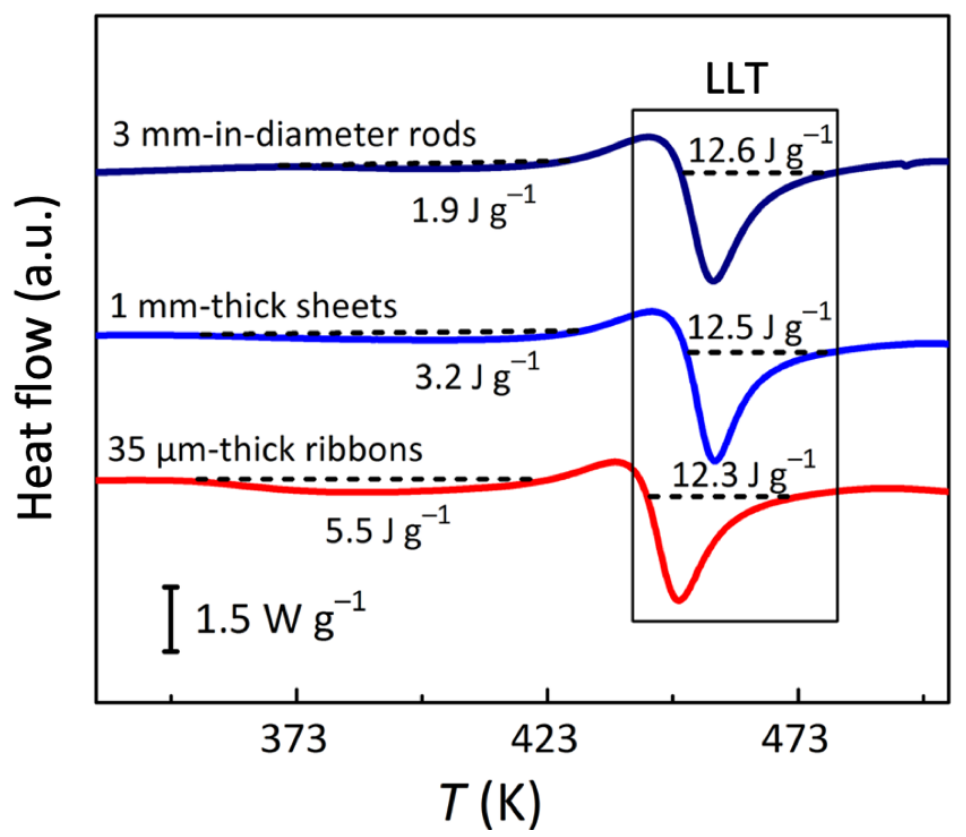

Figure S3. The calorimetry response of the as-cast $\mathrm{La}_{32.5} \mathrm{Ce}_{32.5} \mathrm{Co}_{25} \mathrm{Al}_{10}$ in different casting forms. The as-cast ribbons, $1 \mathrm{~mm}$-thick sheets and $3 \mathrm{~mm}$-in-diameter rods show similar post- $T_{\mathrm{g}}$ exotherm even though the pre- $T_{\mathrm{g}}$ exotherm is different as a result of the effective cooling rate in casting. 

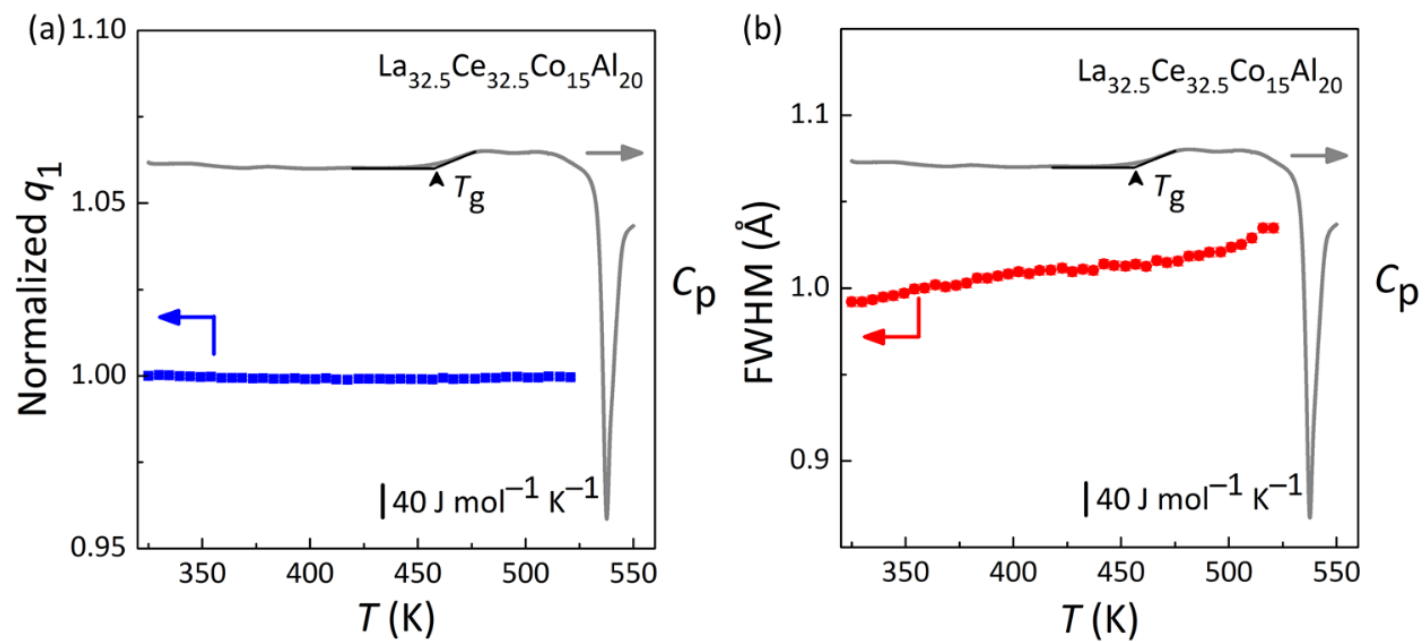

Figure S4. The structure evolution of La32.5Ce32.5Co15Al20 MG during continuous heating. (a) Normalized $q_{1}$ and (b) FWHM of $g(r)$ show no abrupt structural changes in the supercooled liquid state for a usual MG (without LLT). 


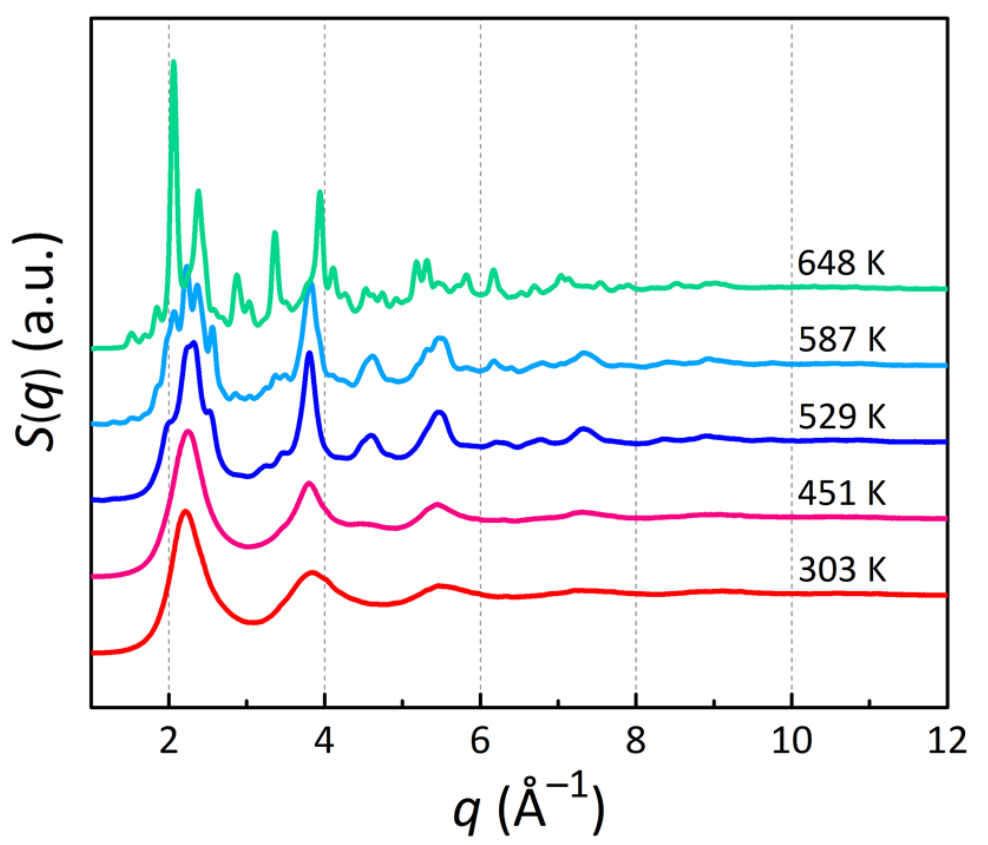

Figure S5. $\boldsymbol{S}(q)$ curves for a sample during in-situ heating. In-situ SXRD shows $S(q)$ curves during heating (heating rate is $10 \mathrm{~K} \mathrm{~min}^{-1}$ ) at representative temperatures. The peak temperatures of four exothermic peaks in DSC are $451 \mathrm{~K}, 529 \mathrm{~K}, 587 \mathrm{~K}$ and 648 $\mathrm{K}$, respectively. 

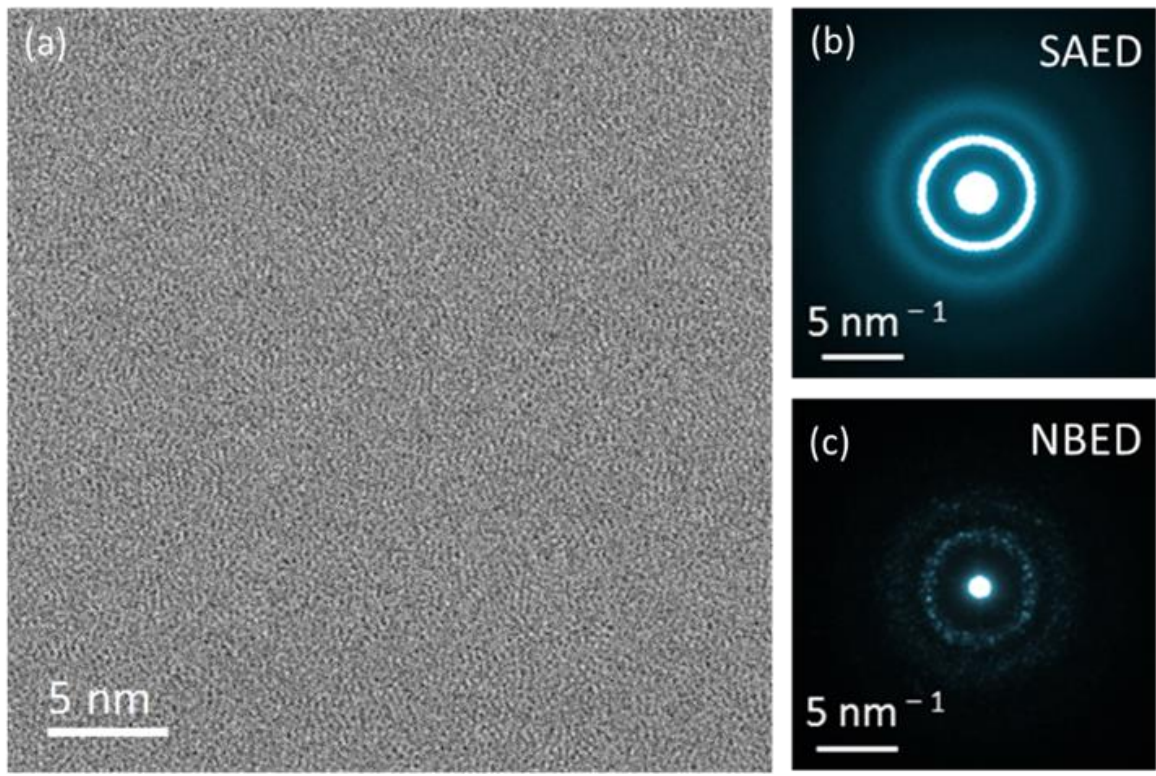

Figure S6. The HRTEM image (a), the corresponding SAED pattern (b) and NBED pattern (c) of the as-cast La32.5 $\mathrm{Ce}_{32.5} \mathrm{Co}_{25} \mathrm{Al}_{10}$ MG. No trace of crystallization is observed in the as-cast sample. 

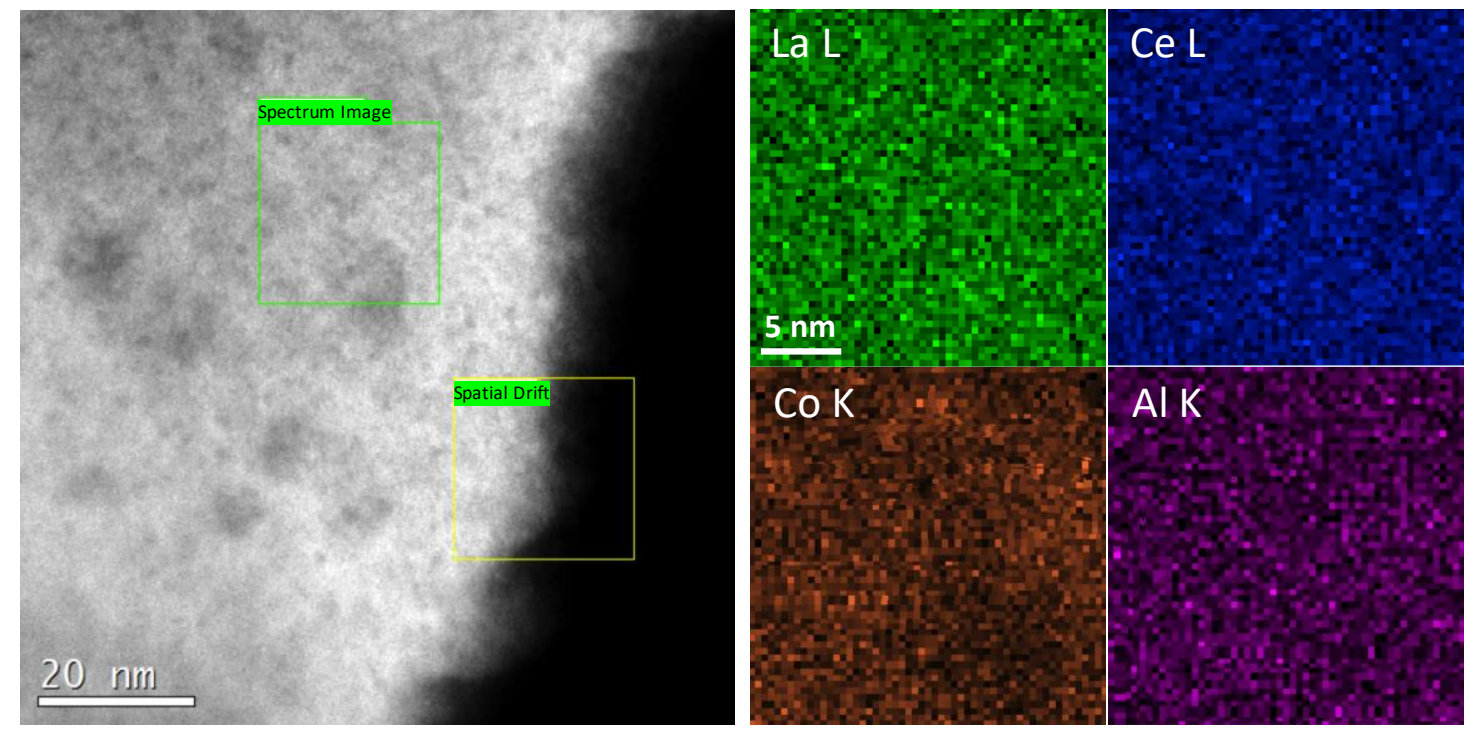

Figure S7. Energy-Dispersive Spectroscopy (EDS) mappings of the 480K-annealed

MGG. No visible chemical variation of $\mathrm{La}, \mathrm{Ce}, \mathrm{Co}$ and $\mathrm{Al}$ are detected at a few nm lengthscale in such a large detective region. Here, we would like to emphasize that below $1 \mathrm{~nm}$, investigation on any potential chemical variation of MGG may lose its physical meaning because below $1 \mathrm{~nm}$, the original MG is chemically heterogeneous. For example, the icosahedron is an energetically favored type of cluster existing in MGs, but the composition of the icosahedrons must be different from that of the nonicosahedron regions, otherwise, the non-icosahedron regions would also form icosahedrons. Therefore, below some critical lengthscales (e.g. $1 \mathrm{~nm}$ ), MG and MGG are both chemically heterogeneous. 

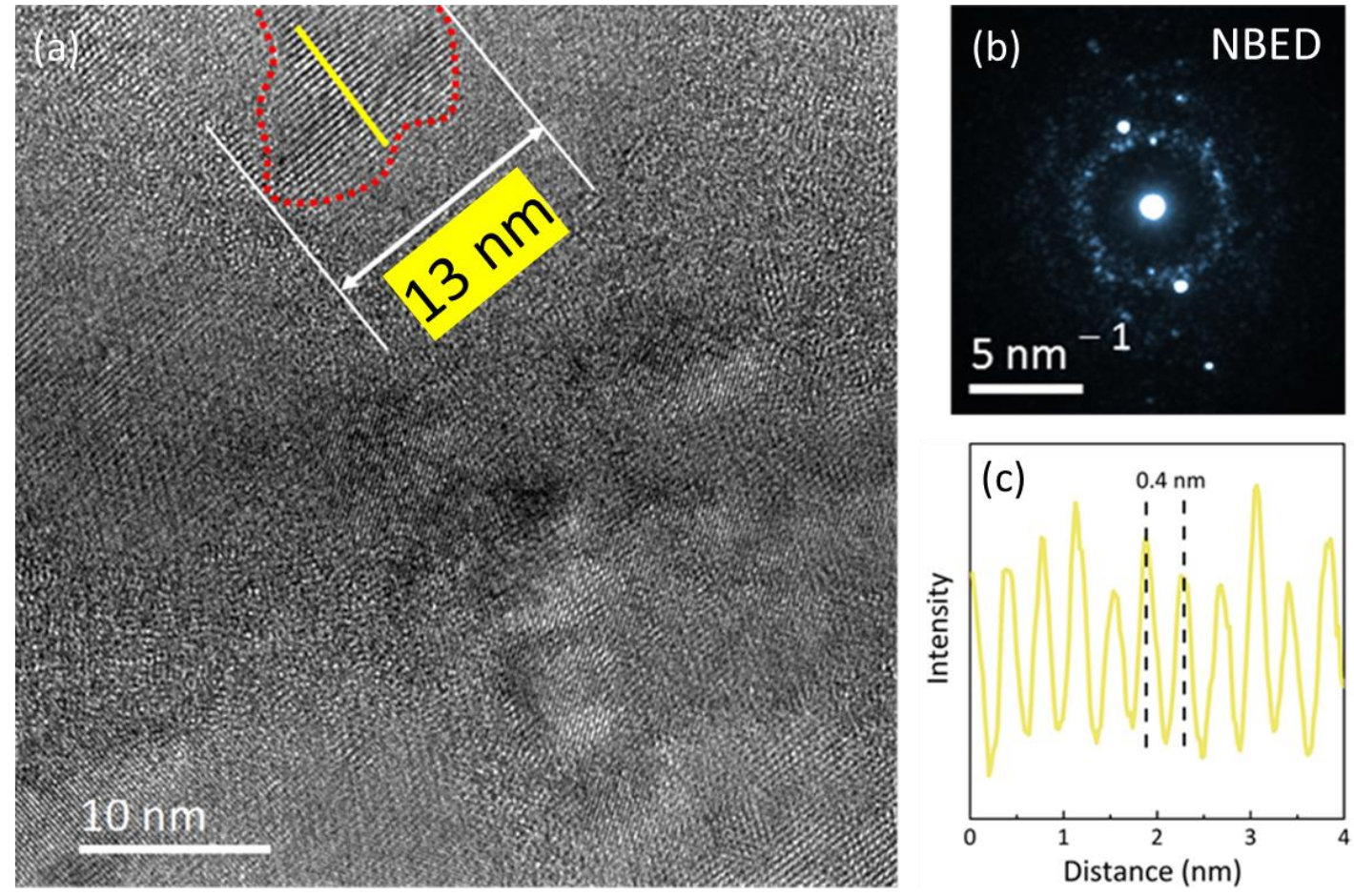

Figure S8. Atomic structure of $550 \mathrm{~K}$-annealed sample (after passing the $\mathbf{2}^{\text {nd }}$ exothermic peak on heating in DSC). (a) nano-crystals $(\sim 10 \mathrm{~nm})$ can be observed in the HRTEM image. (b) Bright points of crystallinities can be observed on the NBED. (c) The pattern of intensity along the yellow solid line in (a), the average distance between two intensity peaks is $0.4 \mathrm{~nm}$, i.e., the interplanar crystal spacing is $0.4 \mathrm{~nm}$. 